\title{
Evangélisation SUR INTERNET
}

\author{
Par le Témoignage de la FOI ${ }^{1}$
}

\section{Dimas Danang Agus Widayanto}

Institut Catholique de Paris, France ddanangaw@gmail.com

\begin{abstract}
:
Since her existence, the Church has assumed her vocation to evangelize through various means of communication. Indeed, many Catholics today, including those in Indonesia, are committed to the proclamation of the Gospel on the internet. How can we effectively evangelize in the digital era? Does communication through the web allow an encounter with Jesus Christ? Delving on these questions, this paper talks about the use of social media platforms (YouTube, Facebook, Instagram, and Twitter) in Indonesia for evangelization. This study, drawing on the works of Antonio Spadaro, Renaud Laby, Guy Marchessault, Jean Ladrière and Paul Ricœur, brings out the effectiveness of the reading of the testimonies of faith that encourage the Christian Internet user to read Biblical texts, so that he can experience his encounter with Jesus Christ. However, it also discovers the existence of the "enclave effects" on the internet that prevent the encounter with "otherness" to become a true encounter. Thus, this paper states that the testimonies broadcasted on the social medias favor rather evangelization ad intra than evangelization ad gentes, which limits the use of the internet to communicate our faith.
\end{abstract}

\section{Keywords:}

Evangelization, Catechesis, Testimony, Internet, Digital Social Media

1 Cet article résume, par l'auteur lui-même, un mémoire rédigé en vue de l'obtention de la licence canonique de théologie spécialisation en théologie catéchétique et pratique soutenu, en Juin 2018, à l'Institut Catholique de Paris, sous la direction d'Isabelle Morel. Ce mémoire est intitulé La performativité des récits de témoignage de la foi, Analyse théologique de l'utilisation des Réseaux Sociaux Numériques en Indonésie pour l'évangélisation. Pour cet article, certaines données de ce mémoire sont mises à jour. 
L'Église est appelée à annoncer la Bonne Nouvelle du Royaume de Dieu (cf. Lc 4,43). En effet, " évangéliser est la grâce et la vocation propre de l'Église. $»^{2}$ Depuis sa naissance, elle assume cette vocation en employant les moyens dont elle a toujours disposé. Voyant les potentialités des nouveaux médias à rendre efficace la communication, elle encourage les chrétiens à se servir de ces moyens pour rendre présent le message du salut de Jésus-Christ. ${ }^{3}$ En citant l'encyclique du Pape Pie XII, Miranda Prorsus (1957), l'instruction pastorale Communio et Progressio (1971) nous enseigne que : «l'Église considère ces moyens de communication comme des 'dons de Dieu' » ( $\left.n^{\circ} 2\right)$. En effet, à l'heure actuelle marquée par l'utilisation massive d'outils numériques, beaucoup de catholiques en Indonésie s'engagent à diffuser et partager les homélies, la prière ou le témoignage. Bref, ils essayent de communiquer l'Évangile en utilisant les Réseaux Sociaux Numériques (RSN). ${ }^{4}$ C'est ainsi que, dans ce pays où les chrétiens sont dispersés et minoritaires, Internet apparait comme la «nouvelle agora $»^{5}$ dans laquelle se réalise « une culture de la rencontre ${ }^{6}$.

\section{Utilisation des RéSeaux Sociaux Numériques}

En Indonésie, selon la statistique publiée par We Are Social en janvier 2019, 56\% de la population utilise Internet. ${ }^{7}$ L'une des caractéris-

2 Cf. Paul VI, Evangelii Nuntiandi, Exhortation Apostolique, 1975, No. 13-14.

Cf. Concile Vatican II, Inter Mirifica, Décret Sur Les Moyens de Communication Sociale, 1963, No. 2-3.

4 Le système d'Internet du type de bibliothèque fixe du web 1.0 est remplacé par celui du web 2.0, qui rend la communication sur la toile plus interactive et participative. Cela fait naître les sites de Réseaux Sociaux Numériques (RSN), habituellement appelé les médias sociaux ou les réseaux sociaux. Ce «web social » progresse ensuite vers le web 3.0 ou le « web sémantique » qui, par une fonction algorithmique, traite automatiquement les bases de données des internautes. C'est par l'utilisation massive des objets connectés qu'apparaît récemment le « web des objets », parfois appelé le « web 4.0 ». (Cf. Véronique Mesguich et Anne-Marie Libmann, Rechercher l'information stratégique sur le web : sourcing, veille et analyse à l'heure de la révolution numérique (Louvain-la-Neuve: De Boeck supérieur, 2018), p. 13.)

5 Benoît XVI, « Message du Pape pour la $47^{\text {ème }}$ Journée Mondiale des Communications Sociales ", 2013. https://w2.vatican.va/content/benedict-xvi/fr/messages/communications/ documents/hf_ben-xvi_mes_20130124_47th-world-communications-day.html (consulté le 31.12.2019).

6 François, « Message du Pape pour la $48^{\text {ème }}$ Journée Mondiale des Communications Sociales », 2014. https://w2.vatican.va/content/francesco/fr/messages/communications/documents/ papa-francesco_20140124_messaggio-comunicazioni-sociali.html (consulté le 31.12.2019).

${ }^{7}$ Cf. We Are Social, “Digital 2019: Indonesia.” https://datareportal.com/reports/digital-2019-in- 
tiques de ces internautes est l'influence importante des RSN, dont 92\% sont des utilisateurs compulsifs : par jour, ils passent 3h26 sur les RSN, une durée plus longue que leur temps à regarder la télévision qui est de 2 h52. En raison d'utilisation massive du smartphone, le nombre d'utilisateurs indonésiens qui consultent les réseaux sociaux en se servant du téléphone portable représente plus de 90\%. Les plateformes des RSN les plus consultés sont YouTube, Facebook, Instagram, et Twitter. Pour Facebook, il y a 130 millions d'utilisateurs indonésiens, dont 44\% de femmes et 56\% d'hommes. Pour Instagram, il y a 62 millions d'utilisateurs indonésiens, dont $49 \%$ de femmes et $51 \%$ d'hommes. Alors que sur Twitter, il y a 6,43 millions d'utilisateurs actifs de l'Indonésie, dont $35 \%$ de femmes et $65 \%$ d'hommes. Parmi les utilisateurs de Facebook et Instagram, 33\% sont des 18-24 ans, 33\% des 25-34 ans, 15\% des 13-17 ans et $12 \%$ des $35-44$ ans.

\section{La présence des catholiques indonésiens sur la toile}

L'Indonésie est un pays laïc dans sa constitution, mais l'adhésion à une religion doit être exprimée publiquement. C'est ainsi que, dans notre recherche, sans beaucoup de difficultés, nous pouvons avoir un aperçu de l'activité numérique des catholiques indonésiens sur Internet. Cette recherche a été réalisée du $1^{\text {er }}$ novembre 2017 au 14 mars 2018 (pour YouTube) et du $1^{\text {er }}$ au 14 mars 2018 (pour Facebook, Instagram et Twitter). Nous avons choisi 25 chaînes de YouTube, 25 pages de Facebook, 25 comptes publics d'Instagram, et 25 comptes publics de Twitter. Ce sont les plus actifs parmi les espaces numériques qui se déclarent appartenant au catholicisme. En employant des outils d'analyse numérique, nous avons enquêté sur leurs publications et interactions, tout en récoltant leurs données publiques. 


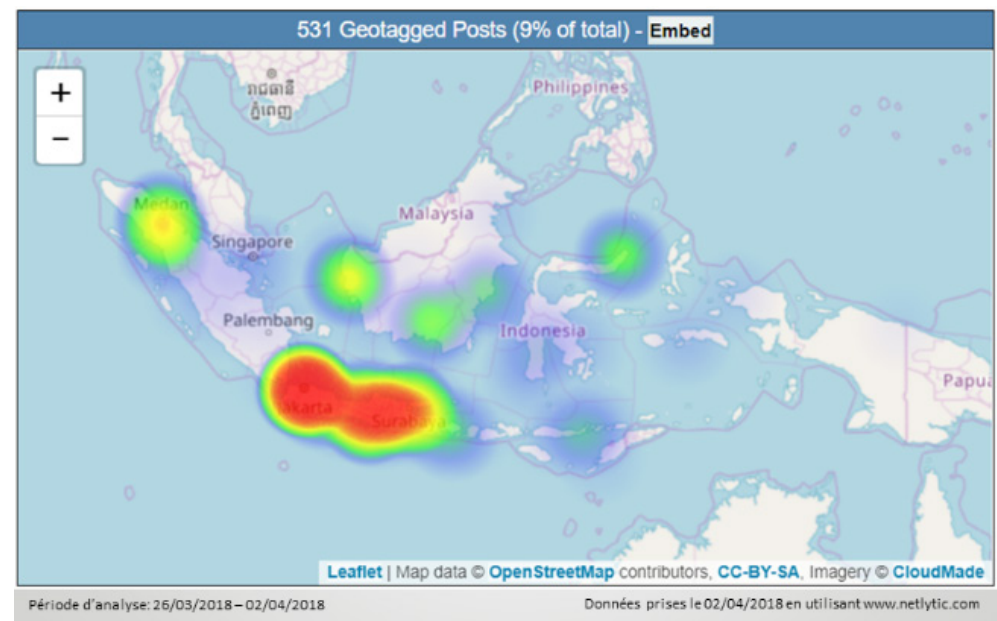

Figure 1 - Recherche par mot-clé « Katolik» sur Instagram

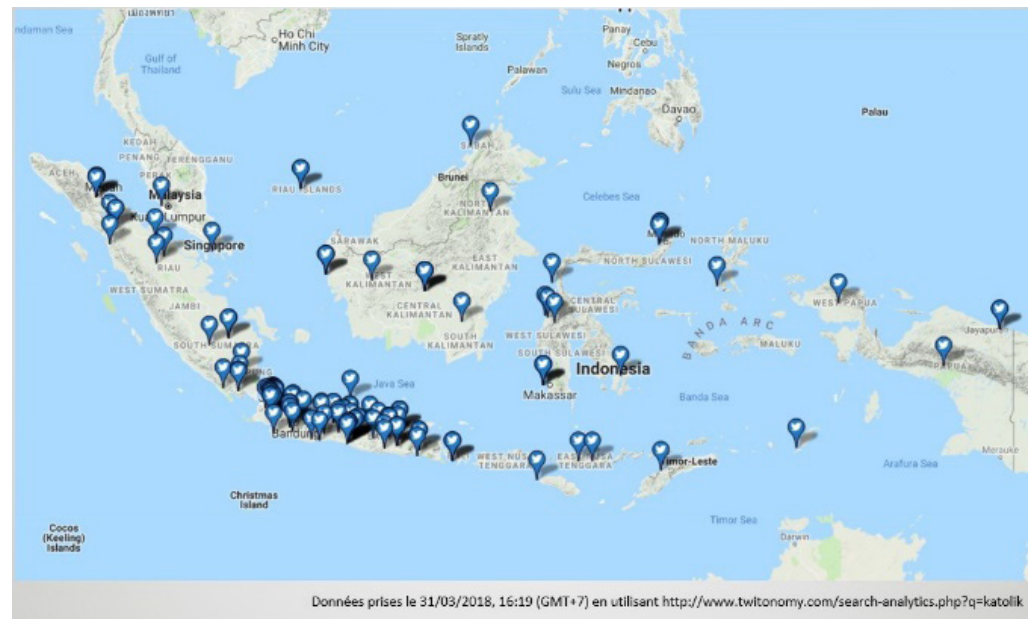

Figure 2-Recherche par mot-clé «Katolik»sur Twitter

Cette recherche quantitative nous amène à comprendre l'activité numérique des catholiques indonésiens. Sur les RSN, il y a une quantité considérable des abonnés de chaînes/pages/comptes qui se déclarent en lien avec le catholicisme. Cependant par rapport du nombre total d'utilisateurs indonésiens, ils ne sont qu'une petite partie. Cela pourrait provenir du fait que le nombre de catholiques indonésiens ne représente que $3 \%$ de la population totale du pays. Par ailleurs, sur un plan géographique (voir figure 1 et 2), notre analyse démontre qu'il existe un grand décalage entre les utilisateurs de l'île de Java avec d'autres régions, de même entre les régions urbaines et les régions rurales. Parmi les espaces numériques analysés, ceux qui sont créés «par une initiative 
personnelle ou par un groupe » sont plus nombreux que "les institutionnels $»^{8}$. Mais quoi qu'il en soit, ces réseaux d'initiative personnelle ou de groupe gardent un lien avec la hiérarchie de l'Église en plaçant un (ou plusieurs) prêtre(s) dans un rôle d'accompagnant ou de consultant.

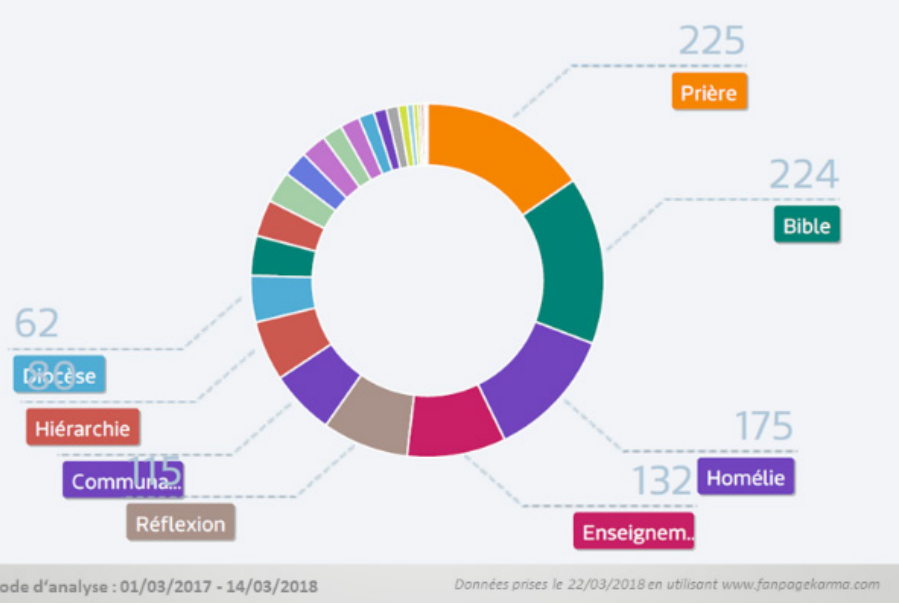

Figure 3 - Nombre de publications sur les pages Facebook analysées, selon leur catégorie

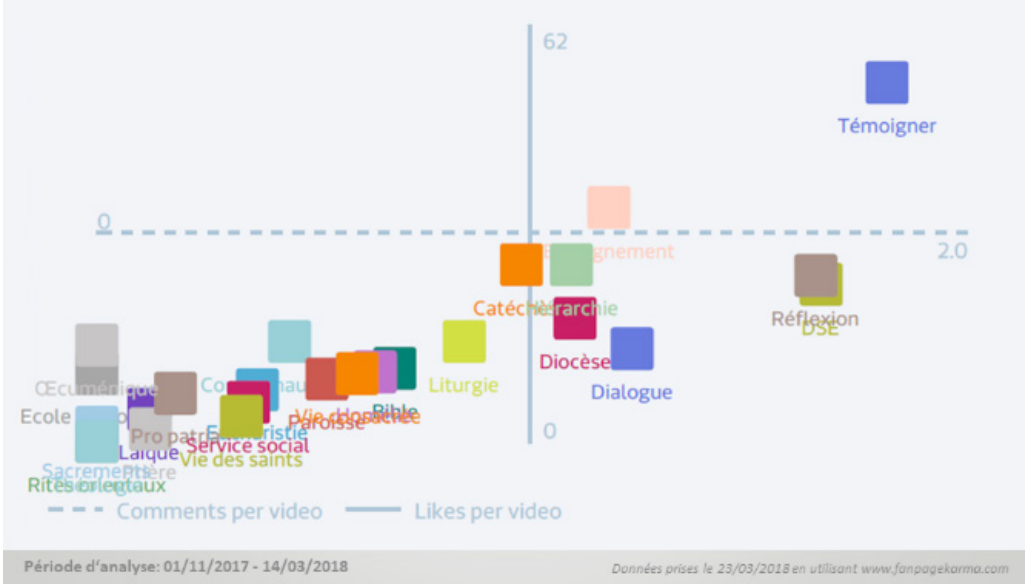

Figure 4 - «J'aime » et commentaires par vidéos publiées sur les chaînes YouTube analysées Le caractère important de la communication sur les RSN est son interactivité : plus une publication suscite d'interactions, plus elle gagne de popularité. Pour observer ce fait, nous avons analysé 3.225 publications : 713 sur YouTube, 852 sur Facebook, 667 sur Instagram, et 993 sur Twit-

8 C'est-à-dire les chaînes/comptes/pages créés par les services diocésains ou paroissiaux, ou bien par les congrégations religieuses. 
ter. Parmi elles, nous avons remarqué la présence importante des publications de type "Enseignement », c'est-à-dire une explication magistrale de la doctrine ecclésiale et de la Bible (voir figure 3). Les publications de type «Témoignage» ne sont pas nombreuses, seulement $3 \%$ du total des publications analysées. Pourtant, il est à noter que le nombre de publications ainsi que le nombre des abonnés des RSN ne sont pas une garantie de nombreuses interactions. En fait, bien que le nombre de publications de type «Enseignement » soit très dominant, ce sont les publications de type «Témoignage » qui déclenchent des interactions vivantes comme les vues, les mentions «J'aime » et les commentaires des internautes (voir figure 4). Parmi celles-là, nous avons remarqué la présence forte du témoignage du pouvoir de la prière, suivi par celui de la vie conjugale, de la vie intérieure et de l'activité ecclésiale.

\section{Défis pastoraux de l'utilisation des réseaux sociaux}

En général, une relation électronique à distance possède quelques spécificités : 1) elle est désincarnée, puisque l'apparence du corps des interlocuteurs est dématérialisée; 2) elle est déterritorialisée, puisqu'elle ne nécessite pas la présence des interlocuteurs dans un espace topographique déterminé; enfin 3) elle peut être désynchronisée, puisque la temporalité de la communication peut être différée bien qu'elle puisse être également instantanée. ${ }^{9}$ Force est de constater que par le processus de transformation des moyens de communication, l'homme et sa culture se transforment également. Certes, un média n'est pas un canal de transmission neutre comme le dit Marshall Mcluhan, un théoricien des médias : "the medium is the message " (le médium, c'est le message). En effet, la communication de la foi intermédiatisée par les RSN relève de nouveaux défis à notre façon de vivre la foi et la vie ecclésiale. Pour le dire autrement, le fonctionnement de ce réseau numérique produit un mode de penser, de communiquer et de réaliser la vie sociale, qui peut affecter l'annonce de l'Évangile. D'abord, la possibilité de jouer avec la présentation de l'identité de chacun suscite la question de la véracité de tous. Ensuite, la proximité d'une relation médiatisée fait douter de la profondeur de la communication et de la qualité de l'information sur ces réseaux, ce qui permet de se demander si l'idéal d'une communication authentique existe. Quoi qu'il en soit, le défi majeur de l'utilisation des RSN est celui de «l'enfermement».

Les RSN piègent l'internaute par deux «effets d'enfermement » : le premier est lié à la nature du réseau social symétrique, et le second par les

${ }^{9}$ Cf. Pierre Mercklé, Sociologie Des Réseaux Sociaux (Paris: La Découverte, 2011), 79. 
algorithmes. ${ }^{10}$ Les RSN ont une nature de réseau social symétrique qui suppose une relation réciproque de sorte qu'on ne voit que la publication et l'information des «amis » déjà acceptés. En conséquence, l'utilisateur se retire dans sa «bulle » avec des «amis » qui lui ressemblent, partagent la même vision et ne remettent jamais en question ses convictions. Cette situation est propice à la circulation massive de fausses informations ou de discours incitant à la haine. En Indonésie, cela apparaissait clairement lors de l'élection du gouverneur de Jakarta en février 2017. ${ }^{11}$ C'est à cause de cette menace pour la vie sociale paisible et la tolérance religieuse dans ce pays que sont intervenues les arrestations, par la police indonésienne, des administrateurs des groupes en ligne accusés de fabriquer des "fake news» ainsi que de propager des discours haineux et des propos diffamatoires sur les réseaux sociaux. ${ }^{12}$ Par ailleurs, en enquêtant sur les publications diffusées sur Twitter de 2006 à 2017, un groupe des chercheurs américains a découvert que les fausses nouvelles étaient retransmises beaucoup plus vite et atteignaient plus d'internautes que la vérité dans toutes les catégories d'information. ${ }^{13}$

C'est par la fonction de déterminer le tissu relationnel des internautes que les algorithmes enferment l'utilisateur des RSN dans sa «bulle». Par exemple, l'algorithme de Facebook, anciennement appelé EdgeRank, détermine la visibilité des publications sur le fil d'actualité (news feed) des utilisateurs en les hiérarchisant basé sur les indicateurs tels que les réactions, les commentaires, et les partages. ${ }^{14}$ Sans être directement identifiable par les internautes, cet algorithme fait un calcul sur leur comportement pour reconnaître des affinités, faire des recommandations, hiérarchiser des contenus, organiser des réponses à une recherche, etc., ce faisant il détermine la manière dont la publication

10 Alexis Delcambre et Alexandre Piquard, «Facebook est-il un danger pour la démocratie ? », Le Monde, http://www.lemonde.fr/actualite-medias/article/2016/11/01/facebook-faux-amide-la-democratie_5023701_3236.html (consulté le 9.5.2018).

11 Merlyna Lim, «Freedom to Hate: Social Media, Algorithmic Enclaves, and the Rise of Tribal Nationalism in Indonesia », Critical Asian Studies 49, No. 3 (July 2017) 411-427.

12 Cf. Koran Tempo, “Jokowi Berang kepada Saracen,” Koran Tempo. Le lundi, 28 août 2017. https: / / koran.tempo.co/read/420940/jokowi-berang-kepada-saracen (consulté le 31.12.2019).

13 Cf. Soroush Vosoughi, Deb Roy, et Sinan Aral, "The Spread of True and False News Online," Science 359, No. 6380 (March 2018) 1146-1151.

14 Adam Mosseri, "News Feed FYI: Bringing People Closer Together," Facebook Business, consulté le 31 décembre 2019, https://www.facebook.com/business/news/news-feed-fyi-bringing-people-closer-together. 
et l'information seront reçues et vues. Par le lien "les amis des amis ", il propose automatiquement des contacts avec lesquels l'utilisateur des RSN peut interagir ou faire "amitié » en ligne. Par ailleurs, tous les internautes ne disposent pas des mêmes ressources sociales et culturelles, c'est pourquoi les algorithmes des RSN reproduisent «les inégalités de nos sociétés en donnant aux mieux dotés les moyens d'enrichir leurs réseaux relationnels et d'accéder à plus de ressources et d'opportunités $»^{15}$.

\section{Problématique théologique et hypothèse}

Nous avons observé que le fonctionnement des RSN enferme l'internaute sur lui-même. Autrement dit, à cause de leur caractère symétrique et de leur système algorithmique actuel, nous doutons que leur communication puisse devenir une authentique culture de la rencontre. En conséquence, leur capacité d'absorption comme média d'évangélisation est remise en question. C'est alors que nous nous demandons si la communication de la foi sur les RSN peut servir à l'évangélisation et conduire à la culture de la rencontre.

Le fait que, sur les RSN, les publications de type "Témoignage " peuvent déclencher une interaction vivante de la foi rejoint l'appréhension qu'à l'heure du numérique l'évangélisation consiste d'abord à témoigner de sa foi. Ainsi, nous nous sommes demandé dans quelle mesure les récits de témoignage diffusés sur les RSN peuvent engendrer la performativité du langage de la foi qui permet la rencontre avec Jésus Christ. Pour y répondre, nous avons fait l'hypothèse selon laquelle la joie de la rencontre personnelle avec le Christ déploierait la créativité des fidèles vivant dans la culture numérique pour témoigner de l'amour de Dieu ; une lecture des récits de témoignage intégrant la dialectique de l'explication et de l'interprétation, permettrait à celui qui reçoit le témoignage de la foi de devenir à son tour, un témoin.

Cette hypothèse nous a conduit à faire deux méthodes de recherche. D'abord, une recherche bibliographique pour éclairer ce qu'est "évangéliser » sur Internet. En considérant qu'évangéliser consiste d'abord à témoigner, nous étudierons les œuvres de Jean Ladrière et de Paul Ricœur pour découvrir ce que signifie le récit du témoignage de la foi et comment il devient porteur de la foi reçue et vécue. Nous réaliserons

15 Dominic Cardon, À quoi rêvent les algorithmes : nos vies à l'heure des big data (Paris: La Seuil, 2015), 67. 
enfin une recherche des pratiques de l'utilisation des RSN par les catholiques indonésiens pour analyser si les récits de témoignage diffusé sur la toile permettent la rencontre avec le Christ.

\section{« ÉVANGÉLISER » SUR INTERNET}

Pour comprendre la notion de l'évangélisation à l'heure du numérique, nous allons étudier les œuvres d'Antonio Spadaro (consultant au Conseil Pontifical pour les Communications Sociales), de Renaud Laby (spécialiste de la question des usages du numérique chez les croyants) et de Guy Marchessault (ancien journaliste puis formateur en communication).

\section{Différents points de vue}

Dans son livre Cyberthéologie, Antonio Spadaro ne se contente pas seulement d'analyser la toile au service de la foi, mais il cherche à saisir le sens profond du net dans le projet de Dieu. Pour lui, Internet est une réalisation des désirs fondamentaux de l'être humain, à savoir le désir de relation et de communication. Ainsi, le net est un contexte existentiel de l'homme dans lequel, pour le christianisme, le défi n'est pas celui du comment bien « utiliser » Internet, mais comment bien "vivre » à l'heure du numérique. Autrement dit, d'un côté considérant la toile comme un milieu où elle vit, l'Église s'adapte de manière critique à la culture numérique ; de l'autre, acceptant les valeurs transmises par l'Église, le net devient un chemin conduisant l'humanité vers Dieu. Spadaro propose ainsi la «cyberthéologie » qu'il définit comme «l'intelligence de la foi au temps d'Internet, c'est-à-dire la réflexion sur la possibilité de penser la foi à la lumière de la logique du net $»^{16}$. Pour lui, le cyberespace, c'est-àdire un espace de communication ouvert par l'interconnexion mondiale des ordinateurs et des mémoires informatiques, est un lieu dans lequel il construit sa réflexion théologique.

En partant d'une critique littéraire, l'analyse de Spadaro se concentre sur la question "comment 'bien' vivre à l'heure du numérique ». Il explique que la caractéristique horizontale et symétrique des RSN ne convient pas à l'image authentique de l'Église. Quand il traite le sujet du moteur de recherche sur Internet, il relève déjà un grand risque du fonctionnement des algorithmes dans sa note de bas de page : celui de rester enfermé dans une "bulle » telle que l'internaute ne puisse

16 Antonio Spadaro, Cyberthéologie : penser le christianisme à l'heure d'Internet (Bruxelle: Lessius, 2014), 33. 
qu'accéder à ce qui correspond à son profil et à ses intérêts. De cette manière, «je serai entouré par un monde d'informations qui me ressemble, risquant ainsi de rester fermé à la provocation intellectuelle qui vient de l'altérité et de la différence. ${ }^{17}$ Après avoir traité ce sujet et ayant constaté ce grand risque, Spadaro ne poursuit pas sa réflexion. C'est pourquoi nous allons approfondir la nôtre en lisant le mémoire de Renaud Laby qui traite d'une manière différente le fonctionnement algorithmique d'Internet.

Dans son mémoire intitulé Internet et communication évangélique, en rendant compte du fait que le fonctionnement technique d'un média produit un mode de pensée affectant l'annonce de l'Évangile, Renaud Laby imagine un dialogue entre la réflexion théologique et la réflexion médiologique. Celle-ci se traduit à une explication sur le fonctionnement des algorithmes qui désigne les limites de l'annonce de la Bonne Nouvelle en ligne. C'est pourquoi Laby constate qu'Internet est moins un outil d'évangélisation qu'un moyen de communication à évangéliser. Il comprend que, comme un processus complexe qui participe de la Révélation, la communication évangélique ne se renferme pas sur ellemême, mais encourage celui de l'altérisation. De ce fait, évangéliser ne se réduit jamais à l'annonce d'un message, mais consiste à témoigner que s'accomplit en elle-même le salut de Dieu qu'elle annonce. Par-là, Laby admet qu'Internet n'est pas simplement un outil à l'extérieur de notre vie mais il est notre milieu dans lequel nous avons toujours besoin d'être évangélisé : «L'Église n'évangélise pas si elle ne se laisse pas continuellement évangéliser » (Evangelii Gaudium No. 174).

Pour Spadaro ainsi que pour Laby, l'évangélisation à l'heure du numérique consiste d'abord à témoigner de sa foi car, «en se révélant à l'humanité, Dieu ne vient pas simplement lui transmettre une information ou un simple savoir sur lui-même, Il l'invite à partager sa vie $»^{18}$, de plus "l'homme du web se fie aux opinions sous forme de témoignage $»^{19}$. Cependant le fait qu'un témoignage favorise un partage de l'expérience personnelle indique son insistance à une orientation plus anthropologique que kérygmatique. De cette manière, considérant que la foi chrétienne n'est pas gravée culturellement dans la vie de beaucoup

17 Antonio Spadaro, Cyberthéologie, 46.

18 Renaud Laby, Internet et La Communication Évangélique (Louvain-la-Neuve-Québec-Paris: Cahiers Internationaux de Théologie Pratique, 2017), www.pastoralis.org, 64.

19 Spadaro, Cyberthéologie, 71. 
d'internautes, on « risque d'éliminer le mystère de Dieu et son altérité constitutive, et de réduire Jésus à un modèle de vie ou à une force d'appoint $»^{20}$. C'est pourquoi nous nous demandons : quelle est la manière de témoigner de la foi qui convient le mieux à la culture du numérique ?

Dans son livre, Médias et foi chrétienne, publiée en 2002, Guy Marchessault, s'inspirant des efforts d'aggiornamento de l'après-concile Vatican II, remarque qu'il existe deux approches pour annoncer l'Évangile. ${ }^{21}$ La première se manifeste dans l'évangélisation ad intra qui conduit à se limiter à l'intérieur de l'Église. Quant à la deuxième, elle s'exprime dans l'évangélisation ad gentes qui permet de s'ouvrir à l'extérieur. C'est cette deuxième approche qui conduira les chrétiens à s'adapter à la culture de leur époque. Dans son autre livre, La foi Chrétienne et le divertissement médiatique, publié en 2007, Marchessault trouve que dans la culture de notre époque, marquée par le divertissement, la narrativité relie l'expression de la foi chrétienne et l'expression médiatique propre au divertissement, particulièrement à travers le récit de témoignage. ${ }^{22}$ En considérant que depuis son origine, la confession de la foi chrétienne se manifeste sous la forme de l'histoire du salut, Marchessault pense que le témoignage authentique des chrétiens atteste la foi reçue et annoncée (portée kérygmatique) ainsi que la foi vécue (portée anthropologique). Pour lui, le témoignage chrétien n'apparaît pas comme un discours intellectuel de la foi. Ce propos rejoint l'invitation du Pape Benoît XVI aux chrétiens de partager la source profonde de leur espérance et de leur joie. ${ }^{23}$ Pour ce pape, un tel partage consiste non seulement dans l'expression explicite de la foi mais aussi dans le témoignage évangélique. Dans le même sens, le Pape François insiste pour que le témoignage chrétien ne se réalise pas avec le bombardement de messages religieux, mais avec la volonté de se donner soi-même aux autres. ${ }^{24}$

20 Denis Villepelet, L'avenir de La Catéchèse (Paris: Les Ed. de l'Atelier/Les Ed. ouvrières, 2003), 37.

21 Cf. Guy Marchessault, Médias et Foi Chrétienne, Deux Univers à Concilier : Divergences et Convergences (Quebec: Fides, 2002), 19-28.

22 Cf. Guy Marchessault, La Foi Chrétienne et Le Divertissement Médiatique: Essai de Théologie Pratique (Québec: Les Presses de l'Université Laval, 2007), 374.

${ }^{23}$ Cf. Benoît XVI, « Message du Pape pour la $47^{\text {ème }}$ Journée Mondiale des Communications Sociales" $"$

${ }^{24}$ Cf. François, « Message du Pape pour la $48^{\text {ème }}$ Journée Mondiale des Communications Sociales ». 


\section{Code source : la Révélation}

Ces trois auteurs articulent de façon complémentaire la même idée : pour mener à bien sa mission évangélisatrice, l'Église doit prendre en compte la culture et le milieu dans lequel elle vit. Pour aboutir à ce résultat dans une situation en pleine mutation, il faut d'abord aller à la source de l'évangélisation qui est la Révélation, un mode de dialogue entre Dieu et les hommes qui culmine dans le mystère pascal de Jésus-Christ.

Prenant la manière dont Jésus communique le Royaume de Dieu, le pape François dit : "La joie de l'Évangile remplit le cœur et toute la vie de ceux qui rencontrent Jésus " (Evangelii Gaudium No. 1). Comme pour les premiers disciples, c'est la rencontre personnelle avec Jésus-Christ qui nous envoie en mission évangélisatrice : après avoir vu Jésus et demeuré avec lui, les premiers disciples vont immédiatement proclamer pleins de joie : "Nous avons trouvé le Messie " (Jn 1, 41). De même, après la rencontre avec Jésus, la Samaritaine témoigne de son expérience résultant que «beaucoup de Samaritains de cette ville avaient cru en Lui à cause de la parole de la femme » (Jn 4, 39). Comme nous le dit St. Jean, ce témoignage a pour but particulier : " ce que nous avons vu et entendu, nous vous l'annonçons, à vous aussi, afin que vous aussi vous soyez en communion avec nous. Et notre communion est communion avec le Père et avec son Fils Jésus Christ» (1Jn 1,3). Rencontrer le Christ, c'est aussi ce que désigne l'objectif de la catéchèse : "de mettre quelqu'un non seulement en contact mais en communion, en intimité avec Jésus-Christ »(Catechesi Tradendae No. 5, DGC No. 80).

La mission de Jésus est plutôt de communiquer le salut de Dieu que de transmettre une information. Autrement dit, la communication du Christ n'est pas simplement une transmission d'information mais une invitation à partager la vie divine. C'est pourquoi évangéliser consiste d'abord, pour l'Église, à témoigner de la foi reçue et vécue en elle-même. Autrement dit, évangéliser à l'heure du numérique signifie avant tout témoigner de l'expérience de la rencontre avec Jésus-Christ, de l'amour de Dieu qui se réalise en nous. Il est à noter que le témoignage des chrétiens ne s'adresse pas à eux-mêmes mais doit contribuer au processus d'altérisation qui conduit à la construction intégrale de chacun et de son rapport à la fraternité universelle.

De l'explication évoquée ci-dessus, nous comprenons que la rencontre personnelle avec le Christ précède toute action évangélisatrice. 
Vivre dans la culture numérique, cette joie de la rencontre avec le Christ déploierait notre créativité pour témoigner de notre foi : "Sortir de soimême pour s'unir aux autres fait du bien. S'enfermer sur soi-même signifie goûter au venin amer de l'immanence » (Evangelii Gaudium No.

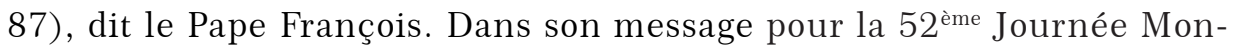
diale des Communications Sociales en 2018 qui critique la propagation des fake news sur les RSN, le Pape François insiste sur le fait que la rencontre avec Jésus-Christ dans laquelle nous nous laissons purifier par Lui, qui est la Vérité ( $c f$. Jn 14,6), est « l'antidote le plus radical au virus du mensonge $»^{25}$. C'est cette expérience de la rencontre avec le Dieu vivant qui libère l'homme : «La vérité vous rendra libres » (Jn 8,32). Ainsi, comme le dit le Pape François : "libération du mensonge et recherche de la relation, voici les deux ingrédients qui ne peuvent pas manquer pour que nos paroles et nos gestes soient vrais, authentiques, fiables. ${ }^{26} \mathrm{C}$ 'est dans ces conditions que la communication de la foi sur les RSN peut servir à l'évangélisation et conduire à la culture de la rencontre.

\section{Récit de Témoignage de la FoI}

Nous avons trouvé qu'évangéliser à l'heure du numérique signifie d'abord témoigner de l'expérience de la rencontre avec Jésus-Christ. C'est ainsi que le récit de témoignage de la foi occupe une place particulière pour l'évangélisation sur la toile. Nous remarquons ici qu'il y a une dimension performative de la communication du Royaume de Dieu. C'est pourquoi, nous allons maintenant approfondir notre compréhension sur la performativité du langage de la foi, particulièrement celle du récit de témoignage de la foi, en étudiant les ouvrages de Jean Ladrière et de Paul Ricœur.

\section{Performativité du langage de la foi}

Jean Ladrière (1921-2007) est l'une des grandes figures de la pensée chrétienne de la seconde moitié du XXe siècle. ${ }^{27}$ Né à Nivelles en Belgique, spécialiste en épistémologie et philosophie des sciences, il est

25 François, «Message Du Pape Pour La 52ème Journée Mondiale Des Communications Sociales », 2018. https://w2.vatican.va/content/francesco/fr/messages/communications/documents/ papa-francesco_20180124_messaggio-comunicazioni-sociali.html (consulté le 31.12.2019).

26 François, « Message Du Pape Pour La 52ème Journée Mondiale Des Communications Sociales».

27 Cf. Hubert Faes, «In Memoriam Jean Ladrière », Transversalites, Vol. 2, No. 106 (2008) 207. 
l'un des pionniers dans les domaines nouveaux de la philosophie contemporaine : analyse du langage et de la culture. Dans son livre, L'articulation du sens, tome II : Les langages de la foi (1984), Ladrière se demande : "malgré la distance historique, $[\ldots]$ comment le langage de la foi est-il assuré d'une continuité d'authenticité dans la variété de ses expressions au cours des temps ? ${ }^{28}$ Il défend l'idée que, dans les textes évangéliques, élaborés au sein de la première communauté chrétienne, se trouve l'expression de l'expérience originaire de la foi. C'est à travers ce qui est dit dans ces textes, en les considérant comme porteurs d'une expérience et en les réactualisant dans leur force attestatrice, que la communauté chrétienne actuelle peut s'approprier l'expérience de la foi de la première communauté et inscrire sa foi dans la sienne. ${ }^{29}$ Dans ce processus, s'implique un cercle herméneutique qualifié de cercle de la foi : « la foi suppose la foi $»^{30}$.

Pour conforter sa thèse, Ladrière met en évidence la pluralité des jeux du langage de la foi. Pour analyser leur particularité, en se fondant sur la théorie des actes de langage ${ }^{31}$, une théorie élaborée par le philosophe anglais J.L. Austin, approfondie par J.R. Searle et Donald Evans, Ladrière élabore son concept de la performativité du langage religieux. Elle se désigne comme une interaction entre la «force illocutoire »d'un énoncé de la foi et son «contenu propositionnel ». ${ }^{32}$ Ladrière définit la «force illocutoire » d'un énoncé de la foi comme le type d'acte qui est réalisé par le fait que cet énoncé est prononcé, et son «contenu propositionnel » comme ce qui est impliqué par l'acte d'énonciation correspondant à la «force illocutoire».

Pour designer la performativité complexe de l'acte de lecture de l'évangile, il propose la notion de " reprise ", c'est-à-dire "l'acte par lequel une communauté réassume pour son propre compte ce qui a été

Jean Ladrière, L'articulation Du Sens, Tome II : Les Langages de La Foi (Paris: Éditions du Cerf, 1984), 11.

29 Cf. Jean Ladrière, L'articulation Du Sens, 8.

30 Jean Ladrière, L'articulation Du Sens, 50 et 53.

31 Cette théorie distingue trois actes dans toute production langagière : «l'acte locutoire » (production d'un énoncé selon un certain nombre de règles linguistiques), « l'acte illocutoire » (intention de l'énonciateur en ce qui concerne le type d'information contenue dans l'énoncé), «l'acte perlocutoire » (effet produit par la production de l'énoncé sur les co-énonciateurs ou sur ses actes). «Illocutionnaire » et «illocutoire » sont synonymes.

32 Cf. Jean Ladrière, L'articulation du sens, 27. 
l'objet d'un témoignage. $\gg^{33} \mathrm{Il}$ distingue deux types de « reprise », à savoir la reprise «superposition » qui s'effectue par une imbrication au moins de deux forces illocutoires sur un contenu propositionnel, et la reprise "réeffectuante » qui reprend à son compte un témoignage de la foi en Jésus. En mettant en évidence les aspects performatifs caractéristiques de chaque variété du langage de la foi, à savoir proclamation, témoignage, attestation, recommandation, présentification, description, etc., Ladrière trouve que le témoignage a une place privilégiée. Il remarque que le mode de témoignage accompagne celui d'attestation. Constatant que la performativité du langage de la foi présuppose la foi, il insiste sur le fait que les croyants de tous les temps gardent leur fidélité créatrice à la foi originaire par ce cercle de la foi. Pour lui, l'attitude propre d'un témoin de la foi est qu'il s'efface devant le contenu de son témoignage, de sorte que ce qui est important, c'est le témoignage lui-même et non le témoin. Il l'explique : "Pour que la Parole de Dieu parle, elle doit passer par un témoignage, mais le témoin s'efface devant la Parole dont il est le porteur, de telle sorte que, dans le témoignage, c'est cette Parole elle-même qui se fait valoir dans sa force propre de révélation et d'interpellation. $»^{34}$

\section{Herméneutique du témoignage}

Paul Ricœur (1913-2005) est l'un des philosophes les plus influents du XXe siècle. Marqué par la philosophie de Husserl et de Heidegger, c'est à lui qu'on doit l'herméneutique contemporaine. Il élabore son article, "L'herméneutique du témoignage ", en dialogue avec la pensée de Jean Nabert. Dans cet article, Ricœur nous fait comprendre le sens et les enjeux des mots "témoin " et "témoignage ", tant dans le langage ordinaire que dans le langage biblique. Il montre que dans le langage ordinaire, le témoignage désigne l'acte de rapporter ce qu'on a vu ou entendu, par la narration ou le récit de l'événement. ${ }^{35}$ Approfondissant le sens quasi-juridique du témoignage, il soulève le problème du faux témoignage qui se focalise plutôt sur la sincérité que sur l'exactitude. Pour lui le témoin s'engage pour la cause qu'il défend, un engagement personnel qui peut conduire jusqu'au sacrifice de sa vie. C'est ainsi que le mot " témoignage » ne désigne pas simplement le rapport d'un témoin oculaire sur

33 Jean Ladrière, L'articulation Du Sens, 51.

34 Jean Ladrière, L'articulation Du Sens, 37.

35 Cf. Paul Ricour, «L'herméneutique du témoignage », in Le Témoignage, Actes Du Colloque (5-11 Janvier 1972), ed. Enrico Castelli (Paris: Aubier-Montaigne, 1972), 38. 
des faits qu'il a vus ou auxquels il a assisté, mais c'est aussi une attestation dans l'action et dans la mort. Par ces engagements, "le témoignage est l'action elle-même [pour autant] qu'elle atteste dans l'extériorité l'homme intérieur lui-même, sa conviction, sa foi. $»^{36}$

Ricœur identifie dans les textes bibliques que le témoignage n'appartient pas au témoin, il vient de l'initiative de Dieu de sorte qu'un témoin est celui qui est envoyé. ${ }^{37}$ Pourtant, cette initiative divine n'éclipse pas la place de l'homme car il n'y a pas de témoin de l'absolu qui ne soit pas témoin des signes historiques. ${ }^{38}$ C'est dans ce sens que le témoignage de la foi implique l'expérience personnelle de la rencontre avec Jésus Christ, rencontre qui nous envoie en mission évangélisatrice. Cette conjonction entre l'initiative divine et l'expérience personnelle nous permet de comprendre la dialectique entre le témoignage et l'attestation. Ce rapport est également évoqué par Jean Ladrière dans Les langages de la foi, quand il définit l'acte fondateur de l'Église primitive comme un témoignage, tandis que la reprise ecclésiale du récit fondateur est considérée comme une attestation. En évoquant ce rapport, ces deux auteurs supposent l'engagement total du témoin, qui distingue le faux témoin du témoin véridique. C'est ainsi que le témoignage désigne une attestation par l'action ou par la mort, définissant que l'engagement de témoignage relie le passé, le présent et le futur du témoin. C'est cette force d'opérativité que Ladrière révèle comme la performativité du témoignage de la foi.

\section{Comment le récit fait-il travailler le lecteur?}

Dans son analyse sur le langage ordinaire ainsi que sur le langage religieux, Ricœur définit le témoignage comme un récit de l'événement. En se souvenant de ce que dit Ladrière, c'est-à-dire que "le langage n'est pas un simple reflet passif de la réalité ou de l'expérience $»^{39}$ nous comprenons qu'un récit de témoignage possède une puissance autonome de construction. Cette puissance présuppose d'un côté un travail chez le témoin, mais d'un autre coté un travail chez le lecteur de sorte qu'à son tour le lecteur devienne aussi un témoin. C'est dans ce cadrage que l'ouvrage de Ricœur, Du texte à l'action, en le replaçant dans l'ensemble

36 Paul Ricœur, «L'herméneutique du témoignage », 43.

$37 C f$. Paul Ricœur, «L'herméneutique du témoignage », 44.

38 Cf. Paul Ricœur, «L'herméneutique du témoignage », 46.

39 Jean Ladrière, L'articulation Du Sens, 71. 
de ses travaux, nous aide à comprendre comment le récit fait travailler le lecteur.

Devant un texte nous sommes, en tant que lecteur, confrontés à deux possibilités : soit «de rester dans le suspens du texte, le traiter comme [un] texte sans monde et sans auteur ; alors nous l'expliquons par ses rapports internes, par sa structure. Ou bien nous pouvons lever le suspens du texte, achever le texte en paroles, le restituant à la communication vivante ; alors nous l'interprétons. ${ }^{40}$ Pour Ricœur, la lecture est essentiellement la dialectique de ces deux attitudes que sont l'explication et l'interprétation. Chacune des deux attitudes renvoie l'une à l'autre par des traits qui leur sont propres. D'un côté, l'analyse structurale récuse une sémantique de surface pour faire apparaître une sémantique profonde. C'est ainsi que l'explication est une étape entre une interprétation de surface et une interprétation en profondeur pour achever la lecture comme une reprise du sens. D'un autre côté, l'interprétation apparaît comme un chemin d'appropriation réalisé par le texte. Par cette dialectique avec l'explication, l'acception subjective de la notion d'interprétation est corrigée de sorte que celle-ci devienne une interprétation objective soulignée par le texte.

De cet ouvrage publié en 1986, nous étudions ainsi la médiation du récit dans laquelle les expériences existentielles pré-narratives s'enchainent d'une façon cohérente par une mise en intrigue, de sorte qu'elle ouvre la projection de tous les possibles qui peuvent être refigurés dans l'expérience actuelle du lecteur. Dans cet enchaînement, le parcours mimétique du récit passe par la préfiguration puis la configuration, et aboutit à la refiguration, ce qui veut dire l'actualisation chez le lecteur. ${ }^{41}$ Ainsi, nous comprenons qu'un bon récit de témoignage de la foi est celui qui laisse au lecteur la possibilité de créer du sens, tout en opérant sur la lecture une dialectique de l'explication et de l'interprétation.

\section{Analyser et InTerpréter les Récits de Témoignage de la Foi}

Dans la lecture des récits de témoignage, la pensée de Ricœur sur la dialectique de l'explication et de l'interprétation est à mettre en parallèle avec celle de Ladrière qui fait l'articulation entre la « force illo-

40 Paul Ricour, «Qu'est-ce qu'un texte ? », in Du Texte à l'action, Essais d'herméneutique II (Paris: Le Seuil, 1986), 145-146.

${ }^{41}$ Cf. Paul Ricour, Temps et Récit Tome I : L’Intrigue et Le Récit Historique (Paris: Le Seuil, 1983), 116117. 
cutoire »d'un énoncé et son « contenu propositionnel». Ainsi, nous considérons que le «cercle herméneutique » se trouve entre notre compréhension et les propositions de sens qui nous sont ouvertes par le récit lui-même. Dans le cadre du langage de la foi, Ladrière appelle ce cercle le « cercle de la foi ", la foi suppose la foi, mais aussi la foi invite à la foi. Pour Ricœur, ce «cercle herméneutique » n'est pas un cercle vicieux. En citant Heidegger, il montre que « l'élément décisif n'est pas de sortir du cercle, mais d'y pénétrer correctement. ${ }^{42}$ C'est pourquoi nous ne faisons l'interprétation du récit du témoignage qu'après avoir analysé structurellement ce récit.

\section{Analyse des rapports internes dans la structure du récit}

Considérant la pluralité des formes des récits de témoignage diffusés sur les RSN, nous les avons classés en trois catégories selon leur manière d'envisager la mise en intrigue, à savoir: le "récit complet » (un film, un dessin animé, une image GIF animée, une vidéo, une publication dans la forme écrite de l'histoire de vie), le "récit visuel fixe " (une image visuelle fixe, une photo, une peinture) et le «petit récit» (les conversations qui accompagnent une publication). Cette classification nous a permis de désigner les critères de l'explication qui découle de l'analyse des rapports internes dans la structure du récit ainsi que les critères de l'interprétation qui conduit à l'appropriation. Pour ce faire, nous nous sommes référés à l'article d'Annik Dubied, "Une définition du récit d'après Paul Ricœur ${ }^{43}$ pour l'analyse du « récit complet ». Lanalyse du « récit visuel fixe » s'est appuyée sur l'article de Raphaël Baroni, "Le récit dans l'image : séquence, intrigue et configuration ${ }^{44}$. Quant à l'analyse du "petit récit », elle prend sa source dans l'article d'Alexandra Georgakopoulou, "Small Stories Research: A Narrative Paradigm for the Analysis of Social Media $»^{45}$.

Pour une lecture du « récit complet », il est suggéré d'analyser les six éléments suivants constituant d'un récit :

42 Paul Ricour, «La tâche de l'herméneutique », in Du texte à l'action, 93.

43 Annik Dubied, «Une définition du récit d'après Paul Ricœur. Préambule à une définition du récit médiatique », Communication, Vol. 2, No. 19 (Février 2000) 45-66.

44 Raphaël Baroni, «Le Récit Dans l'image : Séquence, Intrigue et Configuration », Image \& Narrative, Vol 12, No. 1 (2011) 272-294.

45 Alexandra Georgakopoulou, «Small Stories Research : A Narrative Paradigm for the Analysis of Social Media ", in The SAGE Handbook of Social Media Research Methods, ed. Luke Sloan and Anabel Quan-Haase (London: SAGE Publications, 2017), 266-281. 
i. Un début, un milieu et une fin

ii. Un principe de causalité narrative

iii. Un thème

iv. Une implication d'intérêts humains

v. Une conclusion imprévisible et congruante

vi. Une actualisation

En considérant qu'une image visuelle fixe possède des modalités de la narrativité, l'analyse du "récit visuel fixe » est organisée en trois termes : la séquence, l'intrigue, et la configuration. Plus en détail, ils sont :

i. Séquence

- Insérer une image dans une série (narrativité iconique sérielle)

- Représenter simultanément dans une image les différentes étapes d'un procès (narrativité iconique co-extensive)

- Evoquer sur un mode allusif un récit par une image (narrativité iconique intertextuelle)

ii. Intrigue

- Représenter par une image un procès à un instant critique (suspense iconique)

- Présenter une image ambiguë produisant une synthèse retardée (curiosité iconique)

iii. Configuration : Limage propose une synthèse de l'histoire (configuration iconique)

Sur les RSN, les conversations spontanées se traduisent normalement comme une réponse ou un commentaire à une publication. Nous pouvons considérer la conversation sur les RSN comme une construction du récit par plusieurs personnes. C'est ainsi que la mise en intrigue dans la conversation sur les RSN est une construction commune qui permet à chaque interlocuteur de procéder aux triples mimesis. Pour analyser cette conversation, nous faisons l'analyse du «petit récit » (small stories rechearce). Elle se fait de la manière suivante :

1. Récolter des données des comptes analysés

2. Faire le codage des données

3. Faire l'analyse pour identifier la prise de position narrative et la réécriture 
La prise de position narrative (narrative stancetaking) indique qu'une conversation est offerte ou reprise en tant que récit de sorte que ses participants peuvent se positionner comme narrateur, narrataire, co-narrateur, etc. ${ }^{46}$ Cette activité est ainsi constituée par des événements et des personnages dans un scénario spatio-temporel dont les actions et la parole sont évaluables. Autrement dit, la narrative stancetaking est essentiellement le processus de la mise en récit par l'engagement et la participation des interlocuteurs dans la conversation sur les RSN. La réécriture (rescripting) implique des pratiques des médias permettant de manipuler visuellement et/ou verbalement des publications ou des récits antérieurement publiés afin de créer des récits alternatifs. Ceuxci sont à leur tour offerts et repris comme les récits humoristiques ou satiriques par rapport aux récits originaux. ${ }^{47}$ Autrement dit, la rescripting est le processus de la création d'un récit alternatif basé sur le récit préalablement diffusé.

\section{Critères de l'interprétation qui conduit à l'appropriation}

S'appuyant sur les analyses de chaque type de récit, nous avons progressé pour arriver ensuite à l'appropriation du récit de témoignage. Ici, nous avons défini quelques critères pour que la lecture des récits de témoignage diffusés sur la toile soit fructueuse, c'est-à-dire qu'elle permette la rencontre avec Jésus-Christ, la Parole de Dieu vivant.

Considérant qu'il n'y a pas de témoin de l'absolu qui ne soit pas témoin des signes historiques, nous comprenons le rapport entre l'expérience de la foi et le témoignage. De la logique du "cercle de la foi ", nous pouvons alors penser au "cercle du témoignage ». C'est ainsi que nous nous demandons : de quelle expérience personnelle de la rencontre avec le Christ le témoin nous parle-t-il ? À notre tour, de quelle expérience que pouvons-nous témoigner à autrui ?

Nous avons étudié que la dialectique témoignage-attestation implique l'engagement. Ceci nous invite à interpréter ce récit du témoignage en nous demandant : quelle attestation de la foi ce témoin nous donnet-il ? à quel engagement ce témoin renvoie-t-il ? À notre tour, ce témoignage de la foi nous appelle-t-il à un engagement ?

Nous avons appris l'articulation entre le témoignage de la foi, l'expérience événementielle, et l'engagement. Dans cette articulation, le langage de la foi se rapporte à un eschaton, c'est-à-dire une référence à

46 Cf. Alexandra Georgakopoulou, « Small Stories Research », 275.

${ }^{47}$ Cf. Alexandra Georgakopoulou, « Small Stories Research », 275. 
l'incessant accomplissement et l'incessante attente du salut. ${ }^{48} \mathrm{C}$ 'est dans ce sens que le langage de la foi est un langage d'espérance : il reconnaît la Parole salvifique dans ce qui est annoncé et il affirme sa liaison avec la promesse que cette Parole lui apporte. C'est pourquoi nous pourrons nous demander : quelle est la Parole salvifique, c'est-à-dire la Parole biblique, que ce témoignage reconnaît? dans quelle mesure ce témoignage de la foi fonctionne-t-il pour nous comme un langage d'espérance ?

Nous avons remarqué l'attitude propre d'un témoin de la foi, montrant que celui-ci s'efface devant le contenu de son témoignage, de sorte que ce qui est important c'est le témoignage lui-même et non le témoin. Nous avons étudié également que l'origine et le contenu du témoignage viennent de l'initiative divine de sorte qu'un témoin n'est qu'un envoyé. C'est alors que nous pourrons nous demander : quelle est l'attitude du témoin dans ce témoignage que nous interprétons, sachant qu'une stratégie narrative y est impliquée aussi ? est-ce qu'il a reçu la mission de témoigner de la part de l'Église?

\section{Fécondités et défis du témoignage sur la toile}

Nous avons appliqué les critères et les démarches permettant de faire l'analyse et l'interprétation des récits de témoignage de la foi publiés sur les RSN en Indonésie. Pour ce faire, nous avons pris quelques exemples de récits de témoignage, les avons analysés puis interprétés selon leur manière d'envisager la mise en intrigue, c'est-à-dire selon trois types de récits : "récit complet ", "récit visuel fixe », et "petit récit ». Pour l'analyse du "récit complet ", nous avons pris deux exemples : la vidéo du témoignage de Yuvensia Lidya ${ }^{49}$, publiée sur YouTube, et le texte du témoignage de Yohanes Moh Ridza Abidin ${ }^{50}$, publié sur Facebook. Pour l'analyse du « récit visuel fixe ", nous avons pris l'exemple d'un témoignage photographique : celui d'uno_tiwa_0451. Pour l'analyse du " petit récit », nous avons pris un exemple des commentaires sur la vidéo du témoignage de Daniel Mananta ${ }^{52}$.

48 Cf. Jean Ladrière, L'articulation Du Sens, Tome I : Discours Scientifique et Parole de La Foi (Paris: Aubier-Montaigne - Éditions du Cerf, 1970), 230.

49 Cette vidéo se trouve sur l'adresse https: / / youtu.be/zmhR3I16HcM (consulté le 10.4.2018).

50 Ce témoignage se trouve sur l'adresse https://www.facebook.com/kbundasuci/photos /a.101027183708.114324.100086358708/10154052598228709 (consulté le 10.4.2018).

51 Cette image se trouve sur l'adresse https://www.instagram.com/p/Bf6TJJyBHyk/ (consulté le 11.4.2018).

${ }^{52}$ Cette vidéo se trouve sur l'adresse https: / / youtu.be/mscH_GBkn7A (consulté le 12.4.2018). 
Dans notre recherche qui suit les démarches de l'analyse et de l'interprétation établies particulièrement de la pensée de Ladrière et de Ricœur, nous découvrons la fécondité des récits de témoignage de la foi. Ces démarches peuvent nous conduire à lire notre expérience personnelle à la lumière de la Parole biblique. Ce cheminement permettant de rencontrer le Christ, nous encourage désormais à devenir à notre tour un témoin. Pourtant, il est intéressant de remarquer que les deux tiers des commentaires dans notre analyse de "petit récit », se trouvent être concernés par les sujets de la réécriture qui ont tendance à polémiquer et à jouer avec l'émotion. Des mots durs et des incitations à la violence imprègnent de nombreux commentaires sur les témoignages concernés. Ils ne proviennent pas seulement de la part des internautes qui mettent en question la doctrine chrétienne, mais aussi de la part des commentateurs qui la défendent contre ceux qu'ils considèrent comme adversaires. En fait, les sujets abordés (par exemple, le «péché originel » et la Bible catholique) n'ont rien de nouveau, et il y a quelques sites web catholiques indonésiens qui ont essayé de les expliquer. Constatant aussi le nombre considérable de publications de type de l'enseignement magistral comme nous l'avons évoqué ci-dessus, il est surprenant que dans ces commentaires il n'y ait aucune citation ni référence à ces sites web ou à ces publications.

L'audace et l'engagement des témoins sur Internet méritent d'être salués, pourtant nous remarquons que les conversations concernées sont souvent floues, non vérifiées, et sans référence aux sources fiables. De cette façon, la rencontre avec l'altérité ne devient pas une véritable rencontre, encore moins se transforme-t-elle au service de la fraternité universelle. Ces affrontements avec un point de vue différent n'ont fait que confirmer l'opinion de chacun et intensifier les relations antagonistes que ces internautes cultivaient avec ceux qui sont considérés comme leurs adversaires. Nous avons relevé également que dans toutes nos analyses le statut des témoins comme « un envoyé » de la part de l'Église est toujours en question. Avec la difficulté de le vérifier, nous n'avons pu qu'examiner l'espace numérique sur lequel ce témoignage est publié. C'est pourquoi les "effets de l'enfermement ", algorithmique ou non, et le statut des témoins relèvent les défis particuliers à l'utilisation des RSN pour l'évangélisation. 


\section{Conclusion et Proposition}

La conclusion à laquelle nous sommes arrivés est que la dialectique de l'analyse et de l'interprétation opérée sur notre lecture des récits de témoignage de la foi, nous a fait découvrir la performativité de ces récits. Cette dialectique nous permet de clarifier la "force illocutoire " d'un témoignage pour accéder enfin à son «contenu propositionnel ». Ainsi, la foi reçue et vécue racontée par le témoin dans son récit, permet au lecteur actuel de revivre l'expérience originaire vécue par la première communauté chrétienne, et de la réactualiser afin de l'assumer comme sa propre foi. Autrement dit, intégrant la dialectique de l'analyse et de l'interprétation, nos lectures des récits de témoignage encouragent le lecteur chrétien à lire les textes bibliques de sorte qu'il puisse expérimenter sa rencontre avec Jésus-Christ et devenir à son tour un témoin. Ce constat renforce l'hypothèse que nous avons relatée pour répondre à notre questionnement. Cependant, nous remarquons aussi l'existence des « effets de l'enfermement ", algorithmique ou non, qui empêchent la rencontre avec l'altérité sur les RSN pour devenir une véritable rencontre, encore moins se transformer au service de la fraternité universelle. À partir de là, nous constatons que les récits de témoignage diffusés sur les plateformes de réseaux sociaux favorisent plutôt l'évangélisation ad intra que l'évangélisation ad gentes, ce qui limite l'utilisation de la toile pour communiquer notre foi.

Malgré l'existence des contraintes et des limites, cela ne doit pas empêcher les catholiques d'être " présents en plein cœur de la nouvelle culture des communications $»^{53}$, pour reprendre les mots de Guy Marchessault. Ces défis invitent tous les acteurs de l'évangélisation et tous les hommes de bonne volonté à continuer de proclamer la Bonne Nouvelle de Jésus Christ dans notre culture numérique, dont nous proposons quelques pistes. Nous avons remarqué dans notre recherche le pouvoir du récit dans la construction du sujet. Il est donc nécessaire d'encourager les fidèles à témoigner de leur foi, tant dans la communication en face à face que dans la communication médiatisée sur la toile. Par ailleurs, considérant la performativité du langage de la foi que nous avons observée dans les récits de témoignage diffusés sur Internet, nous pensons que l'utilisation des RSN devrait intégrer la capacité de faire l'herméneutique, c'est-à-dire de faire une lecture en opérant la dialectique de l'explication et de l'interprétation. Pourtant, dans le domaine

53 Guy Marchessault, La Foi Chrétienne et Le Divertissement Médiatique, 374. 
catéchétique, il faut rappeler la limite des récits de témoignage, à savoir que «le témoignage n'est pas identique à la proposition de foi et qu'il ne suffit pas pour faire de la catéchèse. $»^{54}$ Autrement dit, le témoignage ne remplace pas l'acte catéchétique.

\section{Bibliographie}

Baroni, Raphaël. «Le récit dans l'image : Séquence, Intrigue et Configuration. » Image E Narrative, Vol 12, No. 1 (2011): 272-294. http://www.imageandnarrative.be.

Benoît XVI. « Message du Pape pour la 47ème Journée Mondiale des Communications Sociales. » 2013. https://w2.vatican.va/content/ benedict-xvi/fr/messages/communications/documents/hf_ben-xvi_ mes_20130124_47th-world-communications-day.html (consulté le 31.12.2019).

Cardon, Dominic. À quoi rêvent les algorithmes : nos vies à l'heure des big data. Paris: La Seuil, 2015.

Chocarne, Anne-Flore. La communication de Dieu et les nouvelles technologies de l'information et de la communication. Recherches 17. Louvain-laNeuve-Québec-Paris: Cahiers Internationaux de Théologie Pratique, 2016. www.pastoralis.org.

Concile Vatican II. Inter Mirifica, Décret sur les moyens de communication sociale, 1963.

Congrégation pour le Clergé. Directoire général pour la catéchèse (DGC). Bayard-Editions/Centurion/Cerf/Lumen Vitae, 1997.

Conseil Pontifical pour les Communications Sociales. Communio et Progressio, Instruction pastorale sur les moyens de communication sociale, 1971.

Delcambre, Alexis, et Alexandre Piquard. «Facebook est-il un danger pour la démocratie ? » Le Monde.fr, le $1^{\mathrm{er}}$ november 2016. https://www.lemonde.fr/actualite-medias/article/2016/11/01/facebook-faux-ami-dela-democratie_5023701_3236.html (consulté le 9.5.2018).

Dubied, Annik. «Une définition du récit d'après Paul Ricœur. Préambule à une définition du récit médiatique. »Communication. Information médias théories pratiques, Vol. 2, No. 19 (February 2000): 45-66. http://journals.openedition.org/communication/6312.

54 Anne-Flore Chocarne, La Communication de Dieu et Les Nouvelles Technologies de l'information et de La Communication (Louvain-la-Neuve-Québec-Paris: Cahiers Internationaux de Théologie Pratique, 2016), www.pastoralis.org, 37. 
Faes, Hubert. «In Memoriam Jean Ladrière. » Transversalites Vol. 2, No. 106 (2008): 207-210.

François. «Message du Pape pour la 48ème Journée Mondiale des Communications Sociales. » 2014. https://w2.vatican.va/content/francesco/fr/messages/communications/documents/papa-francesco_20140124_messaggio-comunicazioni-sociali.html (consulté le 31.12.2019).

«Message du Pape pour la 52ème Journée Mondiale des Communications Sociales. » 2018. https://w2.vatican.va/content/francesco/fr/messages/communications/documents/papa-francesco_20180124_messaggio-comunicazioni-sociali.html (consulté le 31.12.2019).

Georgakopoulou, Alexandra. "Small Stories Research: A Narrative Paradigm for the Analysis of Social Media. » In The SAGE Handbook of Social Media Research Methods, éd. by Luke Sloan and Anabel Quan-Haase, 266-281. London: SAGE Publications, 2017.

Jean-Paul II. Catechesi Tradendae, Exhortation apostolique sur la catéchèse en notre temps, 1979.

Koran Tempo. "Jokowi Berang kepada Saracen." Koran Tempo. Le lundi, 28 août 2017. Edition No. 5621, Année XVII. https://koran.tempo.co/ read/420940/jokowi-berang-kepada-saracen (consulté le 31.12.2019).

Laby, Renaud. Internet et la communication évangélique. Recherches 18.2. Louvain-la-Neuve-Québec-Paris: Cahiers Internationaux de Théologie Pratique, 2017. www.pastoralis.org.

Lim, Merlyna «Freedom to Hate: Social Media, Algorithmic Enclaves, and the Rise of Tribal Nationalism in Indonesia ", Critical Asian Studies 49, No. 3 (July 2017): 411-427. DOI: 10.1080/14672715.2017.1341188.

Ladrière, Jean. Larticulation du sens, Tome I : Discours scientifique et Parole de la foi. Paris: Aubier-Montaigne - Éditions du Cerf, 1970.

- L'articulation du sens, Tome II : Les langages de la foi. Cogitatio fidei. Paris: Éditions du Cerf, 1984.

Marchessault, Guy. La foi chrétienne et le divertissement médiatique: Essai de théologie pratique. Les Presses de l'Université Laval, 2007.

- Médias et foi chrétienne: Deux univers à concilier: Divergences et Convergences. Quebec: Fides, 2002.

Mercklé, Pierre. Sociologie des Réseaux Sociaux. Repères. Paris: La Découverte, 2011. 
Mesguich, Véronique et Anne-Marie Libmann, Rechercher l'information stratégique sur le web: sourcing, veille et analyse à l'heure de la révolution numérique, Louvain-la-Neuve: De Boeck supérieur, 2018.

Mosseri, Adam. "News Feed FYI: Bringing People Closer Together." Facebook Business. https://www.facebook.com/business/news/news-feed-fyi-bringing-people-closer-together (consulté le 31.12.2019).

Paul VI. Evangelii Nuntiandi, Exhortation apostolique sur l'Évangélisation dans le monde Moderne, 1975.

Ricœur, Paul. Du texte à l'action. Essais d'herméneutique II. Paris: Le Seuil, 1986.

—. "Lherméneutique de Témoignage. » In Le témoignage, Actes du colloque (5-11 Janvier 1972), éd. Enrico Castelli, 35-61. Paris: Aubier-Montaigne, 1972.

—. Temps et Récit Tome I: L'Intrigue et le récit historique. LOrdre philosophique. Paris: Le Seuil, 1983.

Spadaro, Antonio. Cyberthéologie : penser le christianisme à l'heure d'Internet. Trad. Christiane De Paepe. Bruxelle: Lessius, 2014.

Villepelet, Denis. Lavenir de la catéchèse. Interventions théologiques. Paris: Les Ed. de l'Atelier/Les Ed. ouvrières, 2003.

Vosoughi, Soroush, Deb Roy, et Sinan Aral. "The Spread of True and False News Online." Science 359, No. 6380 (March 2018): 1146-1151. DOI : 10.1126/science.aap9559.

We Are Social. "Digital 2019: Indonesia." https://datareportal.com/reports/digital-2019-indonesia?rq=Indonesia (consulté le 31.12.2019).82 